\title{
Tempo de cocção de grãos de feijão em função de doses de fósforo no plantio e do tempo de armazenamento
}

\author{
Juliano Garcia Bertoldo \\ Jefferson Luís Meirelles Coimbra* \\ Altamir Frederico Guidolin \\ Fabiani da Rocha \\ Universidade do Estado de Santa Catarina, Centro de Ciências Agroveterinárias \\ Instituto de Melhoramento e Genética Molecular (IMEGEM) \\ Av. Camões 2090, CEP 88520-000, Lages - SC, Brasil \\ *Autor para correspondência \\ coimbrajefferson@cav.udesc.br
}

Submetido em 01/05/2008

Aceito para publicação em 03/11/2008

\section{Resumo}

Este trabalho teve por objetivo estudar o tempo de cocção em cultivares de feijão cultivado sob diferentes doses de fósforo, ao longo do armazenamento. O experimento foi conduzido na área experimental do Instituto de Melhoramento e Genética Molecular (IMEGEM) da Universidade do Estado de Santa Catarina (UDESC), em Lages, na safra de 2006/07. A densidade de plantas e de semeadura foi de 200.000 plantas por hectare e 15 plantas por metro linear, respectivamente. A unidade experimental foi constituída por seis fileiras de $5 \mathrm{~m}$, espaçadas em $0,5 \mathrm{~m}$, com área útil de $12 \mathrm{~m}^{2}$ por parcela. $\mathrm{O}$ delineamento experimental usado foi de blocos ao acaso, em esquema fatorial $4 \times 3 \times 3$, com três repetições. Quatro genótipos de feijão foram semeados (Pérola, Iapar 81, IPR Uirapuru e IPR Chopim) sendo adicionado três doses de fósforo na linha de semeadura (0, 100 e $200 \mathrm{~kg} . h a^{-1}$ de $\mathrm{P}_{2} \mathrm{O}_{5}$ ). Após a colheita, foram testados três tempos de armazenamento (0, 45 e 90 dias). A avaliação do cozimento dos grãos foi realizada com o uso do cozedor de Mattson, adaptado por Proctor e Watts (1987). A análise de variância revelou um efeito significativo da interação tripla entre os fatores cultivar, doses de fósforo e tempo de armazenamento sobre a variável resposta tempo de cocção. Há uma forte influência do tempo de armazenamento em relação ao tempo de cocção: quanto mais prolongado for o armazenamento, um maior tempo de cocção é necessário. Entretanto, a utilização de adubação fosfatada não foi significativa na redução do tempo de cocção, com exceção do cultivar Iapar 81 tratada com a dose $100 \mathrm{~kg}$.ha-1 de $\mathrm{P}_{2} \mathrm{O}_{5}$ aos 45 dias de armazenamento. Desta forma, tal resultado merece maiores estudos.

Unitermos: Phaseolus vulgaris L., qualidade culinária, cozimento

\section{Abstract}

Effects of different doses of phosphorus during cultivation and length of subsequent storage on the cooking time of beans. This work aimed to verify the cooking times for beans cultivated under different doses of phosphorus and submitted to various periods of storage. The experiment was conducted in the experimental area of Molecular Genetics and Breeding Institute (IMEGEM) at UDESC, in Lages, SC, during the harvest of 
2006/07. The sowing density was 200,000 plants per hectare. The experimental unit consisted of six rows of $5 \times 3 \mathrm{~m}$ in length, $0.5 \mathrm{~m}$ apart, with a useful area of $12 \mathrm{~m}^{2}$ per batch. The experimental delineation employed was that of random blocks in a $4 \times 3 \times 3$ factorial scheme with three repetitions. Four genotypes of beans (Pérola, Iapar 81, IPR Uirapuru and IPR Chopim) were planted and added three doses of phosphorus (0, 100 and 200kg.ha ${ }^{-1}$ of $\mathrm{P}_{2} \mathrm{O}_{5}$ ) in the sowing line. After harvesting, storage times of 0,45 and 90 days were tested. The evaluation of the cooking time of the grains was carried out using a Mattson cooker, adapted by Proctor and Watts (1987). The analysis of variance disclosed a significant effect over the cooking time response for the triple interaction between the factors cultivar (C), dose of phosphorus (P) and time of storage (A) (CxPxA). The time of storage had a strong influence on the time of cooking: the longer the storage, the greater the cooking time required. However, the use of phosphorus for fertilization was not significant in reducing the cooking time, with the exception of cultivar Iapar 81 that was treated with a dose of $100 \mathrm{~kg} \cdot \mathrm{ha}^{-1}$ of $\mathrm{P}_{2} \mathrm{O}_{5}$, followed by storage of 45 days. This significant exception merits further study.

Key words: Phaseolus vulgaris L., culinary quality, cooking

\section{Introdução}

Dentre as leguminosas, o feijão (Phaseolus vulgaris L.) é uma das mais importantes, sendo amplamente cultivado no mundo. O Brasil destaca-se na produção mundial de feijão e também é considerado o maior consumidor, encontrando nessa leguminosa sua principal fonte protéica vegetal (Carbonell et al., 2003). A cultura do feijão tem grande importância no Brasil, sendo consumido em praticamente todos os estados do país, cultivado durante todos os meses do ano e a sua produção provém de quase todo o território nacional. O grande consumo de feijão no Brasil se deve ao aspecto social, econômico e cultural, consistindo de um dos alimentos básicos do povo brasileiro e integrante dos hábitos de consumo de grande parcela da população (Ramos Júnior et al., 2005).

Porém, nos últimos anos, a produção de feijão tem diminuído consideravelmente. Estimativa do IBGE (2007) prevê uma queda de 3,4\% na produção de feijão em grão para a primeira safra de 2007 e para a segunda safra uma queda de $11,1 \%$. Tal queda pode estar relacionada com mudanças na alimentação do brasileiro, devido à falta de disponibilidade de tempo no preparo da alimentação familiar, como ocorria no passado (Ramos Júnior et al., 2005).

Assim, é necessária a investigação de propriedades de cultivares com qualidades tecnológicas superiores desejadas pelos consumidores, como por exemplo, menor tempo de cocção. Um dos principais fatores na preferência de uma determinada cultivar de feijão pelos consumidores e, conseqüentemente, pelos agricultores, está relacionado ao tempo de cocção, isso porque a maioria dos consumidores tem atividades fora do lar e o tempo disponível no preparo das refeições é cada vez menor (Costa et al., 2001).

O tempo de cocção é influenciado por diversos fatores, dentre eles, o tempo de armazenamento. Assim, a preferência do consumidor brasileiro é pelo produto de colheita mais recente, já que a qualidade do feijão é afetada no decorrer do tempo de armazenamento (Esteves et al., 2002).

Com relação aos nutrientes minerais, os grãos de feijão são ricos, principalmente em potássio (25$30 \%$ do conteúdo total de minerais), fósforo (cerca de $0,4 \%$ ), ferro (cerca de $0,007 \%$ ), cálcio, zinco e magnésio (Araújo et al., 1996). Pastorini et al. (2000) relatam que o fósforo é o nutriente que tem proporcionado as maiores e mais freqüentes respostas, sendo que sua baixa disponibilidade no solo afeta negativamente $o$ crescimento das plantas e sua produção.

A relação do tempo de cocção e doses de fósforo no feijão ainda é pouco estudada. O fornecimento adequado e equilibrado de nutrientes para o feijão, como o uso de adubos minerais, poderá contribuir não só para aumentar a produtividade do grão, mas também para melhorar seu valor nutricional (Teixeira et al., 2000). Possivelmente entre as qualidades nutricionais esteja o tempo de cozimento.

Assim, este trabalho teve por objetivo estudar o tempo de cocção em cultivares de feijão com diferentes doses de fósforo ao longo do armazenamento. 


\section{Material e Métodos}

O experimento foi conduzido na área experimental do Instituto de Melhoramento e Genética Molecular (IMEGEM) da Universidade do Estado de Santa Catarina (UDESC), em Lages, situada a $27^{\circ} 47^{\prime} \mathrm{S}$ de latitude, $50^{\circ} 18^{\prime} \mathrm{W}$ de longitude e $916 \mathrm{~m}$ de altitude, na safra de 2006/07. A densidade de plantas e de semeadura foi de 200.000 plantas por hectare e 15 plantas por metro linear, respectivamente. A unidade experimental foi constituída por seis fileiras de $5 \mathrm{~m}$, espaçadas em $0,5 \mathrm{~m}$, com área útil de $12 \mathrm{~m}^{2}$ por parcela.

$\mathrm{O}$ delineamento experimental usado foi de blocos ao acaso, em esquema fatorial $4 \times 3 \times 3$, com três repetições. Os tratamentos foram representados por quatro cultivares de feijão (Pérola, Iapar 81, IPR Uirapuru e IPR Chopim) três doses de fósforo $(0,100$ e 200kg.ha-1 de $\mathrm{P}_{2} \mathrm{O}_{5}$ ) e 3 períodos de armazenamento (0, 45 e 90 dias). Para a adubação, foram aplicadas três doses de fósforo (superfostato triplo $-\mathrm{P}_{2} \mathrm{O}_{5}$ ), $0,100 \mathrm{e}$ $200 \mathrm{~kg} \cdot \mathrm{ha}^{-1}$ na linha de semeadura.

Os tratos culturais, como controle de pragas e plantas invasoras, foram realizados sempre que necessário. Para tanto, foram aplicadas as doses recomendadas $(1 \mathrm{~L} / \mathrm{ha})$ de s-metolacloro, setoxidim (oxima ciclohexanodiona) e metamidofós (organofosforado).

A colheita manual e a trilha das plantas foram realizadas em Janeiro de 2007 e, após uma pré-limpeza, os grãos foram secos em estufa a $26^{\circ} \mathrm{C}$ durante três dias, até atingirem umidade de $12 \%$, em média.

Uma amostra de grãos de cada parcela experimental foi retirada para a determinação do tempo de cocção no tempo zero, sendo o restante dos grãos armazenados em sacos de papel sob temperatura e umidade ambiente durante 45 e 90 dias.

Para o tempo de hidratação, foi utilizada a metodologia proposta por Morris et al. (1950), modificada por Coelho et al. (2007), colocando-se $16 \mathrm{~g}$ de grãos de feijão em erlenmeyers, e adicionado $100 \mathrm{~mL}$ de água tipo I (Direct Q 5 - Millipore), à temperatura de $25^{\circ} \mathrm{C}$, em três repetições. Após uma hora, realizou-se a leitura do volume de água não absorvido pelo grão, bem como o peso dos grãos, e assim sucessivamente até que as amostras estabilizassem. Após o tempo de embebição, foi realizada a avaliação de cozimento dos grãos com o uso do cozedor de Mattson, adaptado por Proctor e Watts (1987). O aparelho é constituído por 25 estiletes verticais, cada um com peso de 90 gramas, terminando com uma ponta de $1 \mathrm{~mm}$ de diâmetro. Os estiletes ficam apoiados sobre os grãos de feijão. $\mathrm{O}$ aparelho, com os 25 grãos, é colocado dentro de uma panela com água destilada fervente (temperatura padronizada de $95^{\circ} \mathrm{C}$ ) e à medida que ocorre o cozimento, os estiletes perfuram os grãos. O tempo de cocção é estabelecido quando 13 estiletes dos 25 perfuram os grãos $(50 \%+1)$.

$\mathrm{O}$ modelo estatístico utilizado neste experimento foi:

$y_{\text {cap }}=m+b=c_{c}+a_{a}+c a_{c a}+p_{p}+c p_{c p}+a p_{a p}+c a p_{c a p}+e_{c a p}$ (Equação 1)

onde:

Fatores experimentais: i) Cultivar (C); ii) Armazenamento (A); iii) Fósforo (P).

Fator de unidade: i) Bloco (B).

Efeitos expressos na equação do modelo estatístico:

m: média; b: efeito do bloco; $\mathrm{c}_{\mathrm{c}}$ : efeito da cultivar; $\mathrm{a}_{\mathrm{a}}$ : efeito do armazenamento; $\mathrm{ca}_{\mathrm{ca}}$ : efeito da interação $\mathrm{CxA} ; \mathrm{p}_{\mathrm{p}}$ : efeito do fósforo; $\mathrm{cp}_{\mathrm{cp}}$ : efeito da interação $\mathrm{CxP}$; $\mathrm{ap}_{\mathrm{ap}}$ : efeito da interação $\mathrm{AxP}$; cap $_{\text {cap }}$ : efeito da interação CxAxR; e:erro;

Os dados foram submetidos à análise de variância pelo teste $F$ global para testar as hipóteses dos efeitos principais e da interação entre os fatores. Quando o efeito da interação foi significativo, os graus de liberdade foram desdobrados, ajustando-se curvas de regressão, através do programa estatístico SAS 9.1.3 (2007).

\section{Resultados e Discussão}

A análise de variância revelou efeito significativo da interação tripla entre os fatores cultivar $(\mathrm{C})$, doses de fósforo (P) e tempo de armazenamento (A) (CxPxA) sobre a variável tempo de cocção (Tabela 1). Segundo Petersen (1977), geralmente o primeiro passo é submeter os dados a uma análise de variância para investigar a existência de diferença significativa entre as médias dos tratamentos. Após a análise de variância, segundo Bertoldo et al. (2008), os graus de liberdade dos 
tratamentos devem ser desdobrados, de acordo com o esquema fatorial adotado, com o intuito de se estudar os efeitos principais dos fatores e os efeitos das interações entre os fatores. Segundo os mesmos autores, o efeito da interação entre fatores é uma medida de variação que ocorre com a característica de estudo correspondente à variação nos níveis de um fator, ao se passar de um nível a outro (efeito simples).

TABELA 1: Análise de variância para a variável tempo de cocção (min) de quatro cultivares de feijão, três doses de fósforo $\left(\mathrm{P}_{2} \mathrm{O}_{5}\right)$ e três tempos de armazenamento. Lages, SC, 2007.

\begin{tabular}{lcc}
\hline Fontes de variação & $\begin{array}{c}\text { Graus de } \\
\text { liberdade }\end{array}$ & Quadrado Médio \\
\hline Bloco & 2 & $64,62^{*}$ \\
Cultivar (C) & 3 & 33,21 \\
Fósforo (P) & 2 & 24,23 \\
Armazenamento (A) & 2 & $1210,73^{*}$ \\
C x P & 6 & 10,10 \\
C x A & 6 & 13,83 \\
P x A & 4 & 0,62 \\
C x P x A & 12 & $38,33 *$ \\
Erro & 70 & 19,12 \\
\hline Total & 107 & - \\
C.V\% & - & 12,38 \\
\hline
\end{tabular}

* Significativo em nível a 5\% de probabilidade de erro pelo teste $\mathrm{F}$.

Assim, para as interações, com efeitos significativos, os graus de liberdade foram desdobrados através do efeito simples, fixando um fator e variando o outro, ou seja: fixando o tempo de armazenamento e variando cultivar e doses de $\mathrm{P}_{2} \mathrm{O}_{5}$ (Tabela 2) e; fixando as doses de $\mathrm{P}_{2} \mathrm{O}_{5}$ e variando a cultivar e o armazenamento (Tabela 3). Para o efeito da interação tripla, os graus do polinômio foram verificados, e posteriormente ajustadas equações de regressão para o efeito do cultivar e doses de $\mathrm{P}_{2} \mathrm{O}_{5}$ sobre o tempo de cocção, fixando-se o período de armazenamento.

De acordo com a Figura 1, ao se fixarem os fatores cultivar e $\mathrm{P}_{2} \mathrm{O}_{5}$, o comportamento do tempo de cocção ao longo do armazenamento apresentou-se linear e semelhante entre as cultivares e doses de $\mathrm{P}_{2} \mathrm{O}_{5}$ exceto para a cultivar Iapar 81 aos 90 dias de armazenamento, em que o comportamento para o tempo de cocção foi quadrático.

TABELA 2: Desdobramento dos graus de liberdade para a interação tripla cultivar versus armazenamento versus doses de $\mathrm{P}_{2} \mathrm{O}_{5}$ $\left(\mathrm{C}^{*} \mathrm{~A}^{* \mathrm{P}}\right)$ para as cultivares Pérola, Iapar 81, IPR Uirapuru e IPR Chopim, fixando o tempo de armazenamento.

\begin{tabular}{|c|c|c|c|}
\hline Cultivar & $\begin{array}{c}\text { Doses de } \mathrm{P}_{2} \mathrm{O}_{5} \\
\left(\mathrm{~kg} \mathrm{ha}^{-1}\right)\end{array}$ & $\begin{array}{c}\text { Graus de } \\
\text { Liberdade }\end{array}$ & $\begin{array}{c}\text { Quadrado } \\
\text { Médio }\end{array}$ \\
\hline Pérola & 0 & 2 & $152,44^{*}$ \\
\hline Pérola & 100 & 2 & $103,00 *$ \\
\hline Pérola & 200 & 2 & $170,33^{*}$ \\
\hline IAPAR 81 & 0 & 2 & $165,77^{*}$ \\
\hline IAPAR 81 & 100 & 2 & $133,77^{*}$ \\
\hline IAPAR 81 & 200 & 2 & $100,33^{*}$ \\
\hline IPR Uirapuru & 0 & 2 & 44,77 \\
\hline IPR Uirapuru & 100 & 2 & $163,44^{*}$ \\
\hline IPR Uirapuru & 200 & 2 & $103,00 *$ \\
\hline IPR Chopim & 0 & 2 & $97,33^{*}$ \\
\hline IPR Chopim & 100 & 2 & $80,44^{*}$ \\
\hline IPR Chopim & 200 & 2 & $168,77^{*}$ \\
\hline Erro & & 70 & 14,96 \\
\hline
\end{tabular}

* Significativo em nível a 5\% de probabilidade de erro pelo teste F.

TABELA 3: Desdobramento dos graus de liberdade para a interação tripla cultivar versus armazenamento versus doses de $\mathrm{P}_{2} \mathrm{O}_{5}$ $\left(C^{*} A^{*} \mathrm{P}\right)$ para as cultivares Pérola, Iapar 81, IPR Uirapuru e IPR Chopim, fixando as doses de $\mathrm{P}_{2} \mathrm{O}_{5}$.

\begin{tabular}{lccc}
\hline Cultivar & $\begin{array}{c}\text { Armazena- } \\
\text { mento (dias) }\end{array}$ & $\begin{array}{c}\text { Graus de } \\
\text { Liberdade }\end{array}$ & $\begin{array}{c}\text { Quadrado } \\
\text { Médio }\end{array}$ \\
\hline Pérola & 0 & 2 & 36,77 \\
Pérola & 45 & 2 & 28,00 \\
Pérola & 90 & 2 & 20,33 \\
IAPAR 81 & 0 & 2 & 0,11 \\
IAPAR 81 & 45 & 2 & $72,33 *$ \\
IAPAR 81 & 90 & 2 & 31,00 \\
IPR Uirapuru & 0 & 2 & 24,11 \\
IPR Uirapuru & 45 & 2 & 15,44 \\
IPR Uirapuru & 90 & 2 & 18,77 \\
IPR Chopim & 0 & 2 & 0,77 \\
IPR Chopim & 45 & 2 & 19,11 \\
IPR Chopim & 90 & 2 & 19,00 \\
Erro & & 70 & 14,96 \\
\hline * Significativo em nível a 5\% de probabilidade de erro pelo teste F.
\end{tabular}


a)
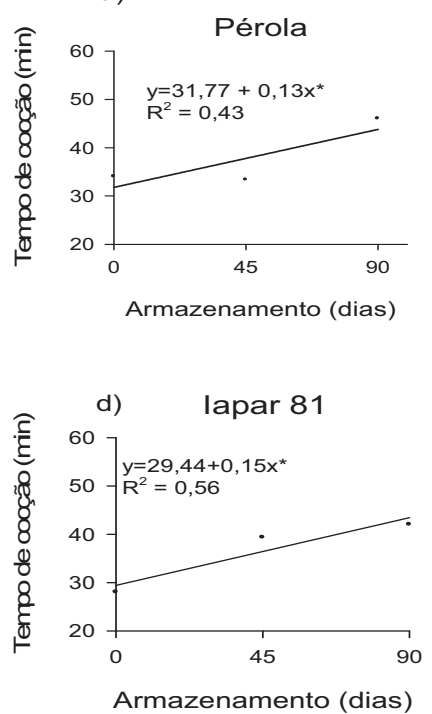

g) IPR Uirapuru
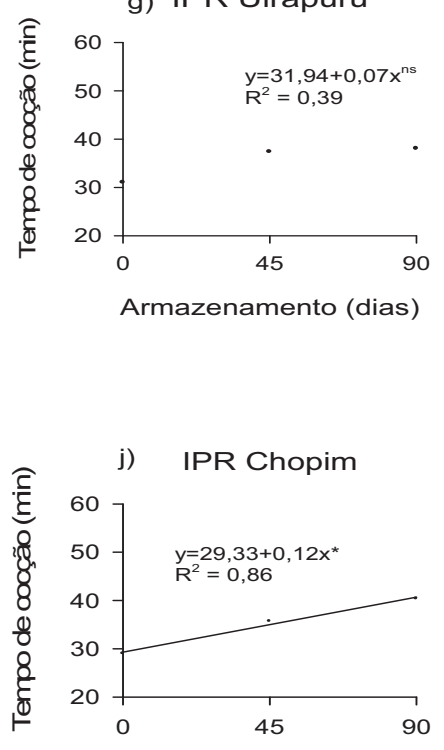

Armazenamento (dias) b)

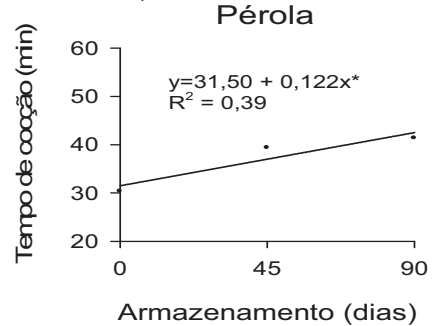

e) Iapar 81

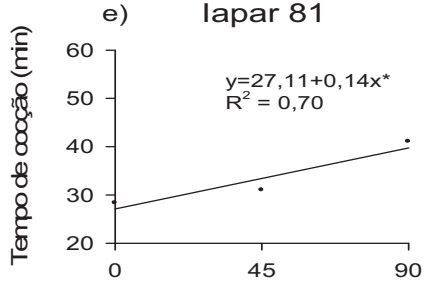

Armazenamento (dias)

h) IPR Uirapuru
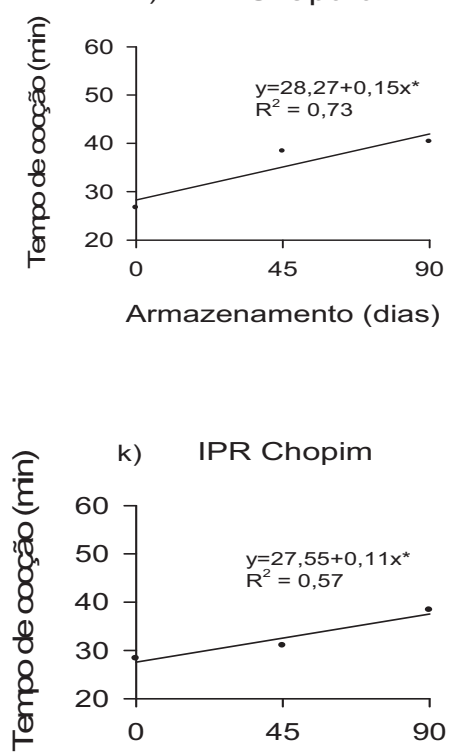

Armazenamento (dias)
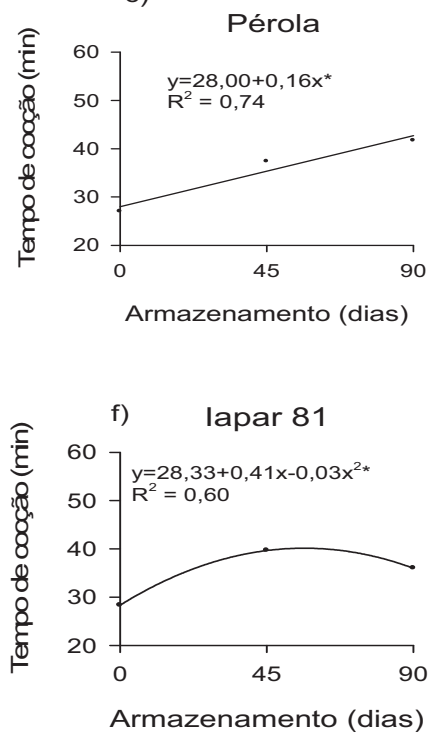

i) IPR Uirapuru

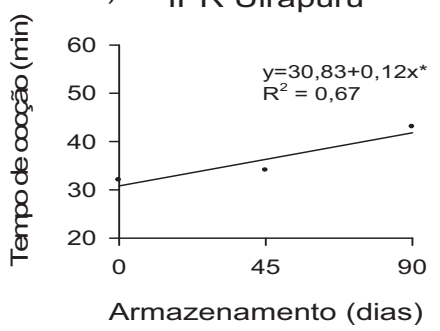

I) IPR Chopim

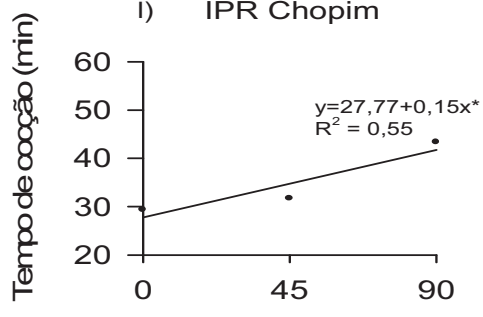

Armazenamento (dias)

FIGURA 1: Comportamento das cultivares combinadas com doses de $\mathrm{P}_{2} \mathrm{O}_{5}\left(0,100\right.$ e $200 \mathrm{~kg}$.ha- ${ }^{-1}$, respectivamente $)$ ao longo do armazenamento para o tempo de cocção. a, d, g, j) sem aplicação de $\mathrm{P}_{2} \mathrm{O}_{5}$; b,e,h,k) 100kg.ha-1 de $\mathrm{P}_{2} \mathrm{O}_{5}$; c,f,i,l) 200kg.ha-1 de $\mathrm{P}_{2} \mathrm{O}_{5}$. 
Para o cultivar Pérola sem a aplicação de $\mathrm{P}_{2} \mathrm{O}_{5}$ (a) houve um aumento de $11,7 \mathrm{~min}$ no tempo de cocção, após 90 dias de armazenamento, enquanto que na dose de $100 \mathrm{~kg} \cdot \mathrm{ha}^{-1}$ de $\mathrm{P}_{2} \mathrm{O}_{5}$ (b) o aumento foi de $10,8 \mathrm{~min}$ e na dose de $200 \mathrm{~kg}$.ha- ${ }^{-1}$ de $\mathrm{P}_{2} \mathrm{O}_{5}$ (c) o aumento foi de 14,4min. O cultivar Iapar 81 sem aplicação de $\mathrm{P}_{2} \mathrm{O}_{5}$ (d) teve um aumento de 13,5min aos 90 dias de armazenamento. Com aplicação de $100 \mathrm{~kg} \cdot \mathrm{ha}^{-1} \mathrm{de}_{2} \mathrm{O}_{5}$ (e), o aumento aos 90 dias foi de 12,6min. Ainda, para o mesmo cultivar com aplicação de $200 \mathrm{~kg}$.ha ${ }^{-1}$ de $\mathrm{P}_{2} \mathrm{O}_{5}$ (f), o ponto máximo foi aos 68 dias de armazenamento, porém aos 90 dias houve um pequeno decréscimo no tempo de cocção, embora ainda maior do que o tempo zero (28,33min). Para o cultivar IPR Uirapuru os efeitos das doses foram significativos somente com a aplicação de $100 \mathrm{~kg} \cdot \mathrm{ha}^{-1}$ de $\mathrm{P}_{2} \mathrm{O}_{5}(\mathrm{~h})$, onde aos 90 dias o aumento na cocção foi de $13,5 \mathrm{~min}$, e na dose $200 \mathrm{~kg}$. ha $^{-1}$ de $\mathrm{P}_{2} \mathrm{O}_{5}$ (i) com um aumento de 10,8min após 90 dias de armazenamento. O cultivar IPR Chopim, sem aplicação de $\mathrm{P}_{2} \mathrm{O}_{5}(\mathrm{j})$ obteve um aumento de $10,8 \mathrm{~min}$ após 90 dias de armazenamento. Na dose 100kg.ha ${ }^{-1}$ de $\mathrm{P}_{2} \mathrm{O}_{5}(\mathrm{k})$, o aumento foi de $9,9 \mathrm{~min}$ aos 90 dias de armazenamento. Ainda, para a mesma cultivar na dose 200kg.ha- ${ }^{-1}$ de $\mathrm{P}_{2} \mathrm{O}_{5}$ (1), o aumento na cocção foi de $13,5 \mathrm{~min}$ aos 90 dias.

De acordo com Rios et al. (2003), a qualidade culinária do feijão é consideravelmente afetada na medida em que se aumentam os meses de armazenamento. Uma das qualidades culinárias afetadas pelo tempo de armazenamento é o tempo de cocção. Rios et al. (2003) relatam que a perda de qualidade se manifesta pelo aumento do grau de dureza do feijão, tendo como conseqüência, o aumento do tempo necessário para o cozimento. $\mathrm{O}$ feijão é um produto que perde rapidamente o valor comercial após a colheita, por causa, principalmente, da diminuição da capacidade de reidratação, do aumento do tempo necessário de cozimento e do escurecimento do tegumento (Bressani, 1993). Segundo Pimentel et al. (1988), avaliando 20 linhagens de feijão, relataram a influência negativa do período de armazenamento em relação ao tempo de cocção, aumentando de menos de duas horas logo após a colheita, para mais de três horas após seis meses de armazenamento.

Durante o armazenamento, ocorrem mudanças fisiológicas nas sementes, principalmente nos cotilédones. O efeito hard-to-cook é resultado de mudanças físicas e químicas que ocorrem nos cotilédones e a níveis intercelulares durante o armazenamento, resultando num aumento da estabilidade da lamela média (Kigel, 1999). Dois fenômenos são responsáveis pelo aumento no tempo de cocção dos grãos de feijão: o desenvolvimento de uma casca dura e alterações nos cotilédones. Tais processos são afetados durante um longo período de armazenamento. Segundo Sgarbieri (1987), as perdas na qualidade do feijão durante o armazenamento são verificadas em virtude do desenvolvimento de casca dura (hardshell) e do escurecimento ou perda das propriedades de cocção dos cotilédones (hard-tocook). Ainda, segundo Hohlberg e Stanley (1987), o tempo prolongado de cocção está diretamente relacionado com dois diferentes processos: hardshell e hard-to-cook. Quando as sementes não absorvem água suficiente, não amolecem durante o cozimento (hardshell). Isto pode estar associado à baixa permeabilidade da casca da semente para hidratar. Por outro lado, mesmo as sementes absorvendo água suficiente o tempo de cocção não diminui (hard-tocook).

A partir dos resultados, pode ser corroborado que não existem diferenças entre os cultivares estudados para o tempo de cocção em relação ao tempo de armazenamento, de modo que, os cultivares testados evidenciaram um comportamento similar do tempo de cocção durante o armazenamento. Resultados similares foram obtidos por Canniatti-Brazaca et al. (1998), os quais observaram que o tempo de cocção aumentou com o tempo de estocagem em todos os cultivares, porém observaram variações entre os locais, e não entre os cultivares. Segundo Coimbra et al. (1999), o componente da interação GxE está altamente relacionado com a cultura do feijão quando cultivado em ambientes diferenciados. Portanto, as diferenças entre os tempos de cocção ao longo do 
armazenamento provavelmente não estão relacionadas com a variabilidade genética das cultivares, mas sim, com fatores de ambiente. Este fato demonstra a dificuldade que os programas de melhoramento podem enfrentar na seleção de constituições genotípicas com menor tempo de cocção. Segundo Costa et al. (2001), há necessidade de se verificar a provável interação cultivares $\mathrm{x}$ ambientes sobre as propriedades culinárias.

Nesse sentido, é de fundamental importância a inclusão de estudos sobre o tempo de cocção em programas de melhoramento, uma vez que é grande a demanda por produtos com elevada qualidade, no intuito de atender as exigências dos consumidores. Assim, o ponto de vista inicial dos melhoristas de feijão, têm se concentrado não somente nos rendimentos das cultivares, resistência a doenças, tolerância a água durante o período de crescimento, mas também na qualidade culinária do grão como demandado pelos consumidores (Wassimi et al., 1990).

Para o tempo de cocção, é mais aceitável que este seja influenciado por fatores de ambiente e/ou fisiológicos, como por exemplo, as condições e o tempo de armazenamento dos grãos e o efeito da interação entre genótipo e ambiente, do que pela adubação mineral. Scholz e Fonseca Júnior (1999) observaram efeitos significativos da interação genótipo $\mathrm{x}$ ambiente em características tecnológicas e culinárias do feijão. De acordo com Dalla Corte et al. (2003) as qualidades tecnológicas e nutricionais dos grãos de feijão são determinadas pelo genótipo e influenciadas pelo efeito de ambiente durante o crescimento da planta e desenvolvimento das sementes. Lange e Labuschagne (2001) mostraram que a interação entre o genótipo e o ambiente pode influenciar significativamente algumas características tecnológicas, como o tamanho da semente, o aspecto visual, a textura, conteúdo de proteínas, o embebimento e quebramento do revestimento das sementes.

Ao se fixar o efeito dos cultivares e do armazenamento e variar o efeito das doses de $\mathrm{P}_{2} \mathrm{O}_{5}$, de acordo com a Figura 2, não se observou efeito significativo para qualquer cultivar ou período de armazenamento, exceto o cultivar Iapar 81 no tempo de armazenamento de 45 dias cujo efeito da interação foi significativo. Houve uma redução no tempo de cocção na dose $100 \mathrm{~kg} \cdot$ ha $^{-1}$ de $\mathrm{P}_{2} \mathrm{O}_{5}$, em relação a testemunha sem aplicação de $\mathrm{P}_{2} \mathrm{O}_{5}$, e posteriormente, aumentando novamente na dose 200kg.ha- ${ }^{-1}$ de $\mathrm{P}_{2} \mathrm{O}_{5}$. Tal fato pode estar indicando que, para o cultivar Iapar 81 aos 45 dias de armazenamento na dose $100 \mathrm{~kg}$. ha ${ }^{-1}$ de $\mathrm{P}_{2} \mathrm{O}_{5}$, há uma redução no tempo de cocção, embora tal resultado necessite de maiores estudos.

Com relação ao fósforo diversos artigos na literatura comentam sobre a importância de sua aplicação sobre o rendimento das sementes. No entanto, a bibliografia é relativamente escassa quanto à utilização do fósforo para se obter melhor qualidade culinária do feijão. Poucos trabalhos relatam sobre a utilização da adubação fosfatada e principalmente, nitrogenada, na melhoria da qualidade tecnológica do feijão. Segundo Teixeira et al. (2000), o fornecimento adequado e equilibrado de nutrientes para o feijão, com o uso de adubos minerais, poderá contribuir não só para aumentar a produtividade do grão, mas também para melhorar o valor nutricional do feijão. Farinelli e Lemos (2005), estudando o efeito de sistemas de manejo do solo e da adubação nitrogenada de cobertura na produtividade, proteína bruta e tempo de cocção do feijão, verificaram que houve uma redução no tempo de cocção, porém pouco vantajoso, concluindo que tal efeito merece maiores estudos.

Assim sendo, há uma influência do tempo de armazenamento sobre o tempo de cocção nos cultivares de feijão estudados, resultando em aumento no tempo de cozimento dos grãos com o passar dos dias de armazenamento. A adubação fosfatada não foi significativa para a redução do tempo de cocção. 


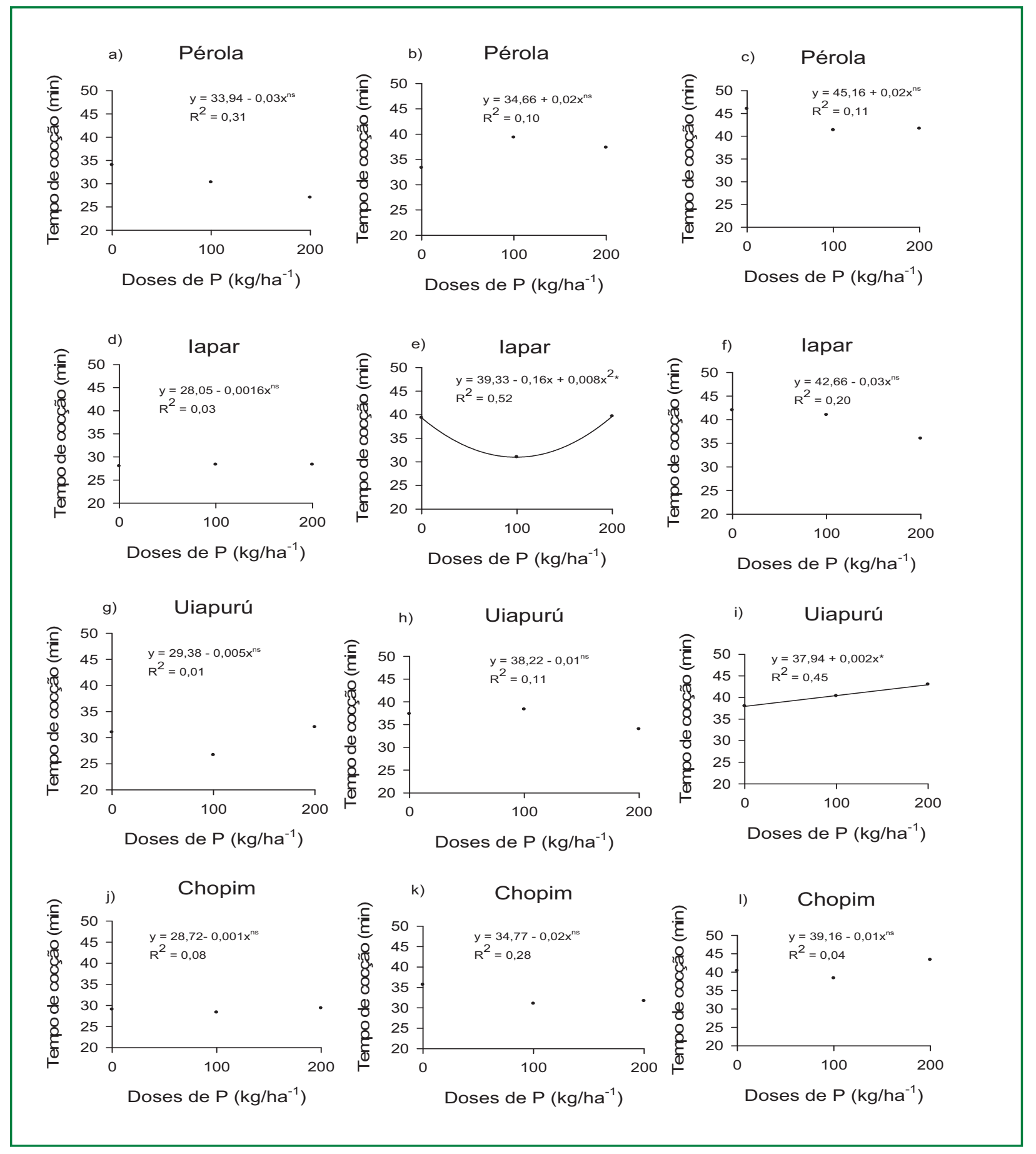

FIGURA 2: Comportamento das cultivares em diferentes tempos de armazenamento (0, 45 e 90 dias) em função de doses de $\mathrm{P}_{2} \mathrm{O}_{5}$ para $\mathrm{o}$ tempo de cocção. a, d, g, j) armazenamento zero; b,e,h,k) 45 dias; c,f,i,l) 90 dias de armazenamento. 


\section{Referências}

Araújo, R. S.; Rava, C. A.; Stone, L. F.; Zimmermann, M. J. O. 1996. Cultura do feijoeiro comum no Brasil. Associação Brasileira para Pesquisa da Potassa e do Fosfato, Piracicaba, Brasil, 786pp.

Bertoldo, J. G.; Coimbra, J. L. M.; Guidolin, A. F.; Mantovani, A.; Vale, N. M. 2008. Problemas relacionados com o uso de testes de comparação de médias em artigos científicos. Revista Biotemas, 21 (2): 145-153.

Bressani, R. 1993. Grain quality of common beans. Food reviews International, 9 (1): 237-297.

Carbonell, S. A. M.; Carvalho, L.; Pereira, V. R. 2003. Qualidade tecnológica de grãos de genótipos de feijoeiro cultivados em diferentes ambientes. Bragantia, 62 (3): 369-379.

Canniatti-Brazaca, S. G.; Mancini Filho, J.; Salgado, J. M.; Novaes, N. J. 1998. Influência do tempo de armazenamento a $11^{\circ} \mathrm{C}$ sobre algumas características físicas de cultivares de feijão guandu (Cajanus cajan L.). Ciência e Tecnologia de Alimentos, 18 (1): 53-59.

Coelho, C. M. M.; Bellato, C. M.; Santos, J. C. P.; Ortega, E. M. M.; Tsai, S. M. 2007. Effect of phytate and storage conditions on the development of the hard to cook phenomenon in common beans. Journal of the Science of Food and Agriculture, 87 (7): 1237 1243.

Coimbra, J. L. M. C; Guidolin, A. F.; Carvalho, F. I. F.; Coimbra, S. M.; Hemp, S. 1999. Reflexos da interação genótipo x ambiente e suas implicações nos ganhos de seleção em genótipos de feijão (Phaseolus vulgaris L.). Ciência Rural, 29 (3): 433-439.

Costa, G. R.; Ramalho, M. A. P.; Abreu, A. F. B. 2001. Variabilidade para absorção de água nos grãos de feijão do germoplasma da UFLA. Ciência e Agrotecnologia, 25 (4): 1017-1021.

Dalla Corte, A.; Moda-Cirino, V.; Scholz, M. B. S.; Destro, D. 2003. Environment effect on grain quality in early common bean cultivars and lines. Crop Breeding and Applied Biotechnology, 3 (3): 193-202.

Esteves, A. M.; Abreu, C. M. P.; Santos, C. D.; Corrêa, A. D. 2002. Comparação química e enzimática de seis linhagens de feijão (Phaseolus vulgaris L.). Ciência e Agrotecnologia, 26 (5): 999-1005.

Farinelli, R.; Lemos, B. L. 2005. Produtividade, proteína bruta e tempo de cozimento de feijão em função de manejos de solo e adubação nitrogenada. Anais do $8^{\circ}$ Congresso Nacional de Pesquisa de Feijão, Goiânia, Brasil, p.854-857.

Hohlberg A. I.; Stanley, D. W. 1987. Hard-to-cook defect in black beans: protein and starch considerations. Journal of Agriculture and Food Chemistry, 1 (35): 571-576.

IBGE. 2007. Sistema IBGE de Recuperação Automática - SIDRA. Disponível em <http://www.sidra.ibge.gov.br/bda/tabela/listabl.asp? $\mathrm{z}=\mathrm{t} \& \mathrm{o}=11 \& \mathrm{i}=\mathrm{P} \& \mathrm{c}=1612>$. Acesso em $01 \mathrm{de}$ junho de 2007.
Kigel, J. 1999. Culinary and nutritional quality of Phaseolus vulgaris seeds as affected by environmental factors. Biotechnologie Agronomie Society Environment, 3 (4): 205-209.

Lange, A. D.; Labuschagne, M. 2001. Multivariate assessment of canning quality, chemical characteristics and yield of small white canning beans (Phaseolus vulgaris L.) in South Africa. Journal of the Science of Food and Agriculture, 81 (1): 30-35.

Morris, H. J.; Olson, R. L.; Bean, R. C. 1950. Processing quality of varieties and strains of dry beans. Food Technology, 4 (3): 247251.

Pastorini, L. H.; Bacarin, M. A.; Lopes, N. F.; Lima, M. G. S. 2000. Crescimento inicial de feijoeiro submetido a diferentes doses de fósforo em solução nutritiva. Revista Ceres, 47 (270): 219-228.

Petersen, G. R. 1977. Use and misuse of multiple comparison procedures. Agronomy Journal, 69 (2): 205-208.

Pimentel, M. L.; Miranda, P.; Costa, A. F.; Miranda, A. B. 1988. Estudo nutricional de linhagens de feijão comum (Phaseolus vulgaris L.). Revista Brasileira de Sementes, 10 (1): 55-65.

Proctor, J. R.; Watts, B. M. 1987. Development of a modified Mattson bean cooker procedure based on sensory panel cookability evaluation. Canadian Institute of Food Science and Technology, 20 (1): 9-14

Ramos Júnior, E. U.; Lemos, L. B.; Silva, T. R. B. 2005. Componentes da produção, produtividade de grãos e características tecnológicas de cultivares de feijão. Bragantia, 64 (1): 75-82.

Rios, A. O.; Abreu, C. M. P.; Corrêa, A. D. 2003. Efeito da estocagem e das condições de colheita sobre algumas propriedades físicas, químicas e nutricionais de três cultivares de feijão (Phaseolus vulgaris L.). Ciência e Tecnologia de Alimentos, 23 (suplemento): 39-45.

SAS (2007). SAS 9.1.3 (TS1M3) for Windows Microsoft. SAS Institute Inc., Cary, USA, sem paginação.

Scholz, M. B. S.; Fonseca Júnior, N. S. 1999. Efeito de ambientes, dos genótipos e da interação genótipos $\mathrm{x}$ ambientes na qualidade tecnológica de feijão do grupo de cores no Estado do Paraná. Anais

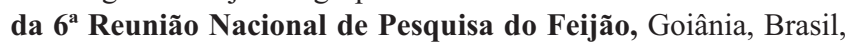
p.339-342.

Sgarbieri, V. C. 1987. Alimentação e nutrição: fator de saúde e desenvolvimento. $1^{\text {a }}$ ed. Editora da Unicamp, Campinas, Brasil, $387 \mathrm{pp}$.

Teixeira, I. R.; Andrade, M. J. B.; Carvalho, J. G.; Morais, A. R.; Corrêa, J. B. D. 2000. Resposta do feijoeiro (Phaseolus vulgaris L. cv. Pérola) a diferentes densidades de semeadura e doses de nitrogênio. Ciência e Agrotecnologia, 24 (2): 399-408.

Wassimi, N. N.; Hosfield, G. L; Uebersax, M. A. 1990. Inheritance of physico-chemical seed characters related to culinary quality in dry bean. Journal of the American Society for the Horticultural Science, 15 (1): 492-499. 